\title{
SYMMETRY BREAKING VIA INTERNAL GEOMETRY
}

\author{
ANDREW TALMADGE \\ Received 15 November 2004 and in revised form 2 July 2005
}

Gauge theories commonly employ complex vector-valued fields to reduce symmetry groups through the Higgs mechanism of spontaneous symmetry breaking. The geometry of the internal space $V$ is tacitly assumed to be the metric geometry of some static, nondynamical hermitian metric $k$. In this paper, we consider $G$-principal bundle gauge theories, where $G$ is a subgroup of $U(V, k)$ (the unitary transformations on the internal vector space $V$ with hermitian metric $k$ ) and we consider allowing the hermitian metric on the internal space $V$ to become an additional dynamical element of the theory. We find a mechanism for interpreting the Higgs scalar field as a feature of the geometry of the internal space while retaining the successful aspects of the Higgs mechanism and spontaneous symmetry breaking.

\section{Introduction}

In this paper, we consider $G$-principal bundle gauge theories, where $G$ is a subgroup of $U(V, k)$ (the unitary transformations on the internal vector space $V$ with hermitian metric $k$ ) as models of particle interactions that employ a complex vector space $V$-valued field, known as a Higgs field. Through the process of spontaneous symmetry breaking, the Higgs field allows the theory to predict masses of bosons and to reduce the principal bundle to an appropriate vacuum subbundle with group $H \subset G([2,11,13])$. In the range of this vector-valued field, there is a canonical hermitian metric $k$. This metric is tacitly chosen to be a constant nondynamical element of the theory.

In this paper, we allow the hermitian metric on the internal space $V$ to become a dynamic element of the theory and we find a way to realize the Higgs scalar field as a feature of the geometry of the internal space while retaining the successful aspects of the Higgs mechanism and spontaneous symmetry breaking. This interpretation is obtained using a Lagrangian procedure to identify a nonzero vacuum value for a special class of internal metric fields. By choosing such a vacuum field, we achieve a symmetry-breaking phenomena similar to those found in the standard theory of electroweak interactions: the prediction of two massive boson fields and one massless boson field.

In addition, the interpretation of the Higgs scalar field as a feature of the geometry of the internal space puts Yang-Mills theories into closer alignment with the general 
relativity theories of Einstein in the following sense. Normally in general relativity dynamical roles are assigned to both the metric and the connection on space time while in Yang-Mills theory, only the connection is a dynamical element of the theory (see $[5,9])$.

Considerations of dynamical internal metrics may be found in earlier works as a supersymmetric theory (see $[1,4])$. Attempts to interpret the internal metric geometrically in Yang-Mills theories also appear in a number of places (e.g., see $[5,7,8]$ ).

The form for the variation of the hermitian metric fields presented here is similar to those found in nonlinear sigma models (see [10]) where a left-invariant Riemannian metric structure on the homogeneous space $G / H$ is employed. This author has presented similar concepts of geometric interpretations of Higgs scalar fields as varying sesquilinear forms in the leptonic sector of the Glashow-Weinberg-Salam model of electroweak interactions (see [12]).

This paper is organized in the following manner. Section 2 contains background material on gauge theories and symmetry-breaking phenomena. Section 3 explains the main result, that is, how metric fields on the internal spaces may be used to break symmetry for a gauge theory. Section 4 discusses the dynamics of the internal metric field and compares these results to the standard theory of electroweak interactions. The last section is a summary of the results contained in this paper.

\section{Background and notation}

A G-gauge theory consists of a principal fiber bundle $\pi: P \rightarrow M$ with Lie group $G$, where $M$ is a four-dimensional space-time manifold. That is, $M$ is a real four-dimensional smooth manifold equipped with a semi-Riemannian metric which has signature $(+,-$, $-,-)$. Unless otherwise noted, all manifolds are $C^{\infty}$ and all maps are assumed smooth.

Suppose that the Lie group $G$ acts on the left of a vector space $V$ and that this action is both effective and faithful. We will indicate this action by $g \cdot v$, where $g \in G$ and $v \in V$. The set of equivariant mappings from $P$ to $V$ is denoted by

$$
C_{G}(P, V)=\left\{\tau: P \longrightarrow V \mid \tau(p g)=g^{-1} \cdot \tau(p)\right\}
$$

Here we have written the right action of $G$ on the principal bundle $P$ as $R_{g}(p)=p g$.

In this paper, we also assume that $V$ is a complex vector space of complex dimension $n$ and the existence of a constant hermitian metric $k$ on $V$. For this paper, we also assume that the gauge group $G \subset U(V, k)$, where

$$
U(V, k)=\{a \in \operatorname{End}(V) \mid k(a(v), a(w))=k(v, w) \forall v, w \in V\}
$$

Particle interaction models (e.g., quantum electrodynamics, electroweak interactions) are typically presented as a Lagrangian field theory on the base space of a principal fiber bundle (see [2]). A gauge transformation is a bundle mapping $f: P \rightarrow P$ such that the diffeomorphism $\hat{f}: M \rightarrow M$ given by $\hat{f}(\pi(p))=\pi(f(p))$ is the identity mapping on $M$. Particle fields are represented by equivariant mappings from the principal bundle into a vector space $V$ upon which $G$ acts on the left. The vector space $V$ with its associated group action is called the "internal space" of the gauge theory. 
In many models of particle interactions, the symmetry group for one type of interaction is contained as a subgroup of the symmetry group for some larger class of interactions. As an example, the symmetry group for the standard model of quantum electrodynamics is isomorphic to $U(1)$ and this group is contained as a subgroup of the symmetry group $S U(2) \times U(1)$. Particle interaction theories which are built in this manner employ a mechanism called "spontaneous symmetry breaking" to select or define a subgroup $H$ of the total symmetry group $G$. In this mechanism, a vector-valued field is introduced. The dynamics of this particle are determined by a Lagrangian sector which includes a potential energy function. This function is chosen so that when one chooses a stable equilibrium point to define the vacuum energy, the remaining symmetries are precisely the subgroup $H$.

To make all of the above discussion more precise, we give the following definition of a symmetry-breaking map (see [13]).

Definition 2.1. $\tau \in C_{G}(P, V)$ is called a symmetry-breaking map if the range of $\tau$ is a single group orbit of the action of $G$ on $V$.

If $\tau$ is a symmetry-breaking map and $\tau_{0}$ is some point in the range of $\tau$, then $Q=$ $\tau^{-1}(V) \subset P$ is a subbundle with structure group $H \subset G$, where

$$
H=\left\{h \in G \mid h \cdot \tau_{0}=\tau_{0}\right\}
$$

We employ the terminology "internal geometry" to mean the choice of an equivariant mapping from the principal bundle $P$ into the space of hermitian metrics (possibly degenerate) on $V$. Equivalently, we can formulate this as a hermitian fiber metric of the vector bundle associated to $P$ via the action of $G$ on the vector space $V$. The internal geometry is typically chosen by using the equivariant mapping having constant value which equals a constant hermitian metric on $V$.

\section{Symmetry-breaking metric fields}

In this section, we show that by choosing an internal geometry which is nonconstant, we may obtain a symmetry-breaking map. Throughout this section, let $\left\{e_{i}\right\}$ denote the standard basis of the vector space $V$ and let $\left\{e^{j}\right\}$ be its dual cobasis. The standard hermitian metric is then given by the form

$$
k=k_{a \bar{b}} e^{a} \cdot \overline{e^{b}}
$$

where "." denotes the symmetric product of the covectors.

Let $\operatorname{Herm}(V)$ be the vector space of (possibly degenerate) hermitian metrics on $V$. Thus, $h \in \operatorname{Herm}(V)$ if (i) $h(v, w)$ is linear in $v$, where $v, w \in V$; (ii) $h(v, w)=\overline{h(w, v)}$; and (iii) $h(v, v) \geq 0$ for $0 \neq v \in V$.

Consider the standard action of $G$ on $\operatorname{Herm}(V)$ induced by the standard action of $G$ on $V$, that is,

$$
\left(g^{-1} \cdot h\right)(v, w)=h(g \cdot v, g \cdot w)
$$


for all $h \in \operatorname{Herm}(V)$ and $v, w \in V$. As defined above, $C_{G}(P, \operatorname{Herm}(V))$ is the set of equivariant mappings from $P$ into $\operatorname{Herm}(V)$.

We may now think of the standard metric employed in particle interaction theories as the map $\delta \in C_{G}(P, \operatorname{Herm}(V))$ which is defined by $\delta(p)=k=k_{a \bar{b}}$ (the constant hermitian metric on $V$ ) for all points $p \in P$.

The standard hermitian metric on $V$ induces a metric $\tilde{k}$ on the set of hermitian metrics $\operatorname{Herm}(V)$ in the following manner. For $r, s \in \operatorname{Herm}(V)$, we have

$$
\tilde{k}(r, s)=k^{i \bar{j}} k^{l \bar{m}} r_{i \bar{m}} s_{l \bar{j}},
$$

where $r=r_{a \bar{b}} e^{a} \cdot \overline{e^{b}}$ and $s=s_{a \bar{b}} e^{a} \cdot \overline{e^{b}}$. Henceforth, we will dispense with the above notation and write $s=s_{c \bar{d}}$. We make the observation that if $\sigma, \rho \in C_{G}(P, \operatorname{Herm}(V))$, the quantity $\tilde{k}(\rho(p), \sigma(p))$ is constant along the fibers of $P$, and hence projects down to a function on the base manifold $M$.

Let $\varphi \in C_{G}\left(P, V^{*}\right)$, where we write $\varphi(p)=\varphi_{a}(p) e^{a}$. In this paper, we will consider perturbations of the standard metric field of the form

$$
\rho=\rho_{a \bar{b}}=c^{2} k_{a \bar{b}}-\overline{\varphi_{a}} \varphi_{b}
$$

where $c$ is a positive real parameter. Using the observation above for $\rho \in C_{G}(P, \operatorname{Herm}(V))$, we define a smooth function called the action density, $L(\rho)(\pi(p))=\tilde{k}(\rho(p), \rho(p))$.

For a perturbation of the form given in (3.4), a straightforward computation shows that

$$
\tilde{k}(\rho, \rho)=n c^{4}-2 c^{2} k(\varphi, \varphi)+(k(\varphi, \varphi))^{2}
$$

Here $k=k^{a \bar{b}}$, where $k^{a \bar{b}} k_{b \bar{c}}=\delta_{c}^{a}$, is also used to denote the hermitian metric on $V^{*}$ induced by the metric on $V$. Using the notation $k(\varphi, \varphi)=\|\varphi\|^{2}$, we may rewrite (3.5) as

$$
\tilde{k}(\rho, \rho)=n c^{4}-2 c^{2}\|\varphi\|^{2}+\|\varphi\|^{4} .
$$

Equation (3.6) is precisely of the form of action density that appears in the standard theory of electroweak interaction (see $[2,11,13])$.

For an open set with compact closure $U \subset M$ and some $\rho \in C_{G}(P, \operatorname{Herm}(V))$, we define the action of $\rho$ over $U$ as

$$
A_{U}(\rho)=\int_{U} L(\rho)(\pi(p)) d m
$$

where $d m$ is the volume form on the space-time manifold $M$ (see [2]).

If we consider minimizing this action over all elements $\rho \in C_{G}(P, \operatorname{Herm}(V))$ of the form $\rho=\rho_{a \bar{b}}=c^{2} k_{a \bar{b}}-\overline{\varphi_{a}} \varphi_{b}$, we will say that $\hat{\rho}$ is stationary relative to the action density $L$ if for all $U \subset M$ (as above) and for all $\sigma \in C_{G}(P, \operatorname{Herm}(V))$ with projected support in $U$, we have

$$
\left.\frac{d}{d t} A_{U}(\hat{\rho}+t \sigma)\right|_{t=0}=0
$$


A straightforward calculation shows that $\hat{\rho}$ is a stationary point if $\|\phi\|^{2}=c^{2}$. We call the choice of such a minimizing element $\hat{\rho}$ a vacuum point, and the set $\{g \cdot \hat{\rho} \mid g \in G\}$ a vacuum orbit. Furthermore, this reduces the symmetry group $G$ to the unbroken subgroup given by

$$
H=\{g \in G \mid g \cdot \hat{\rho}=\hat{\rho}\}
$$

Next if we define $S_{c}^{n}\left(V^{*}\right)=\left\{v \in V^{*}:\|v\|^{2}=c^{2}\right\}$, we can summarize our result as follows. The equivariant hermitian fields that are stationary relative to the action density $L$ are of the form given in (3.4) above with the added restriction that $\varphi$ is a smoothly varying $S_{c}^{n}\left(V^{*}\right)$-valued function on $U \subset M$. This action density selects a new hermitian form at each point in our bundle $P$. For this hermitian form on the internal space, we have vectors $\psi=\lambda \varphi$ such that

$$
\hat{\rho}(\psi, \psi)=0
$$

Hence, we see that the geometry of the internal space is not the standard hermitian geometry of a static space, rather it contains some form of internal "null" vectors which correspond to the choice of the vacuum.

\section{Dynamics of the internal hermitian form}

In the standard theory of electroweak interaction, the Higgs field is a dynamic element of the theory. The Lagrangian sector that provides the field equations for this particle is made up of two parts. One of these is essentially (3.6) above and the other is given by

$$
L(\varphi, \omega)=(\eta k)\left(D^{\omega} \varphi, D^{\omega} \varphi\right)
$$

where $(\eta k)$ is the concatenation of the metric $k$ chosen for the internal space $V$ and the Minkowski metric $\eta$ (here $D^{\omega}$ is the covariant derivative with respect to a connection $\omega$ on $P$ ).

A natural analog for our discussion would be to have a Lagrangian sector given by

$$
L^{\prime}(\rho, \omega)=(\eta \tilde{k})\left(D^{\omega} \rho, D^{\omega} \rho\right)
$$

For the Lagrangian density given in (3.6) and (4.2), we find (following [2]) that the field equation for $\rho$ is given by

$$
\delta^{\omega} D^{\omega} \rho+\rho=0
$$

In $(4.3), \delta^{\omega}$ is the covariant codifferential defined by $\delta^{\omega}={ }^{*} D^{\omega *}$, where $\bar{*}$ is the unique extension of the lift of the star operator to the horizontal subspaces of $P$ that vanishes on vertical vectors (see [2]). For $\rho$ of the form given in (3.4), we may rewrite the field equations in terms of $\varphi$ as

$$
\delta^{\omega} D^{\omega}\left(\overline{\varphi_{a}} \varphi_{b}\right)+\overline{\varphi_{a}} \varphi_{b}=-c^{2} k_{a \bar{b}}
$$


Equation (4.4) reveals that the previously defined constant hermitian acts as a source term for the field equations that $\varphi$ must satisfy.

As a specific example, we return to the standard model case where $G=S U(2) \times U(1)$ and $V=\mathbb{C}^{2}=\left\{\left[\begin{array}{l}v_{1} \\ v_{2}\end{array}\right], v_{1}, v_{2} \in \mathbb{C}\right\}$ with standard hermitian form $\delta=\delta_{a b}$. An element of $G$ is a pair $\left(\begin{array}{cc}\alpha & \beta \\ -\bar{\beta} & \bar{\alpha}\end{array}\right) \in S U(2)$ and $e^{i \theta} \in U(1)$, where the action of $G$ on $V$ is given by $\left(\left(\begin{array}{cc}\alpha & \beta \\ -\bar{\beta} & \bar{\alpha}\end{array}\right), e^{i \theta}\right)\left[\begin{array}{c}v_{1} \\ v_{2}\end{array}\right]=\left[\begin{array}{c}e^{i \theta}\left(\alpha v_{1}+\beta v_{2}\right) \\ e^{i \theta}\left(-\bar{\beta} v_{1}+\bar{\alpha} v_{2}\right)\end{array}\right]$. In this case, we consider the basis for the Lie algebra of $G$ given by $\left\{\sqrt{-1} \tau_{k}\right\}$, where

$$
\tau_{0}=\left(\begin{array}{ll}
1 & 0 \\
0 & 1
\end{array}\right), \quad \tau_{1}=\left(\begin{array}{ll}
0 & 1 \\
1 & 0
\end{array}\right), \quad \tau_{2}=\left(\begin{array}{cc}
0 & -\sqrt{-1} \\
\sqrt{-1} & 0
\end{array}\right), \quad \tau_{3}=\left(\begin{array}{cc}
1 & 0 \\
0 & -1
\end{array}\right) .
$$

For a choice of vacuum $\hat{\rho}$, in the standard theory (working over Minkowski space time) with a local section $s: U \subset M \rightarrow P$, we may write

$$
\hat{\rho}(s(m))=\left(\begin{array}{ll}
c^{2} & 0 \\
0 & 0
\end{array}\right)
$$

by choosing our section so that $\varphi(s(m))=\left[\begin{array}{ll}0 & c\end{array}\right] \in \mathbb{C}^{2^{*}}$, where $c$ is a real parameter. Expressing the field $\varphi^{\prime}$ as a perturbation from the vacuum, we may write using the transpose for the dual vectors $\varphi^{\prime}(s(m))=\left[\begin{array}{ll}0 & c+\varepsilon(m)\end{array}\right] \in \mathbb{C}^{2^{*}}$. This gives us a perturbation from the vacuum for the internal metric as

$$
\hat{\rho}^{\prime}=\left(\begin{array}{cc}
c^{2} & 0 \\
0 & c^{2}-\|c+\varepsilon\|^{2}
\end{array}\right)
$$

Expressing the covariant derivative of the perturbation from the vacuum in local coordinates, we have

$$
D_{\mu} \hat{\rho}^{\prime}=\partial_{\mu} \hat{\rho}^{\prime}+\frac{\sqrt{-1}}{2} g A_{\mu}^{k}\left[\tau_{k}, \hat{\rho}^{\prime}\right]
$$

where $[a, b]=a b-b a$ is the induced action of the Lie algebra on the hermitian form on the internal space.

For our choice of perturbation, we have from (4.2) that

$$
\begin{aligned}
L^{\prime}(\rho, \omega)= & (\eta \tilde{k})\left(D^{\omega} \rho, D^{\omega} \rho\right) \\
= & 4\|c+\varepsilon\|^{2} \eta^{\mu \nu}\left(\partial_{\mu}\|c+\varepsilon\| \partial_{\nu}\|c+\varepsilon\|\right) \\
& +\frac{1}{2} g^{2}\|c+\varepsilon\|^{4} \eta^{\mu \nu}\left(A_{\mu}^{1} A_{\nu}^{1}+A_{\mu}^{2} A_{\nu}^{2}\right)
\end{aligned}
$$

So after expanding the field as a perturbation from the vacuum, we can recognize two massive vector bosons corresponding to the fields $A_{\mu}^{1}$ and $A_{\mu}^{2}$ with masses given by $m=$ $g c^{2}$. The two other vector bosons remain massless and there is no predicted scalar boson or Higgs field. 
We note that unlike the standard theory of electroweak interactions, we have no need for the concept of a Weinberg angle to decouple the electromagnetic field from the Higgs field. This leaves us with the question of the identification of the Lie algebra generator of the photon field. Notice that because we are working with $G \subset U(V, k)$ and we have chosen to work with perturbations of the form given in (3.4), we have a vacuum subgroup given by

$$
H=\{g \in G \mid g \cdot \hat{\rho}=\hat{\rho}\}=\{g \in G \mid g \cdot \varphi=\varphi\}
$$

This subgroup is $H=\left\{e^{\sqrt{-1} \theta Q} \cdot \varphi=\varphi\right\}=\left\{\left(\begin{array}{cc}e^{-\sqrt{-1} \theta} & 0 \\ 0 & e^{\sqrt{-1} \theta}\end{array}\right), e^{\sqrt{-1} \theta}\right\} \cong U(1)$ and this is the subgroup of the Lie subalgebra generated by $Q=\tau_{0}-\tau_{3}$.

\section{Conclusions}

In this paper, we have shown that the formalism of spontaneous symmetry breaking may be modeled as the choice of a special type of hermitian form on the internal space of the theory. An action density which measures the norm of the hermitian form is similar to the one employed by the Higgs mechanism. This new hermitian form defines an "internal geometry" that has internal "null" vectors. These internal "null" vectors correspond to the choices of vacuum points for the theory.

This dynamic choice of the internal metric makes the Yang-Mills theory look more like the general relativity theory of Einstein by making both the metric and the connection in the Yang-Mills theory dynamic elements of the theory.

In addition, we have demonstrated for the standard model of electroweak interactions based on the gauge group $G=S U(2) \times U(1)$ that using a Lagrangian procedure with Lagrangian given by

$$
L(\rho)+L^{\prime}(\rho, \omega)=\tilde{k}(\rho, \rho)+(\eta \tilde{k})\left(D^{\omega} \rho, D^{\omega} \rho\right)
$$

we can obtain a symmetry-breaking phenomena and massive vector bosons similar to the predictions of the standard theory.

It is of interest to note that others have used hermitian forms with similar structure to the ones employed in this paper. In [3], the author describes canonical metrics on holomorphic bundles in which there is a global section. The results here yield the same functionals that arise in the Yang-Mills theory. Metrics generated by scalar fields, using techniques like those given above, have also been used in quark bag models (see [6]).

One shortcoming of this work is that it does not include Lagrangian terms for the leptons; we hope to examine this feature of the theory in future work.

\section{Acknowledgments}

The author would like to thank Professor Larry Norris for suggesting the initial idea of considering the internal geometry of gauge theories. The author is also indebted to the referee for improving this work. 


\section{Symmetry breaking via internal geometry}

\section{References}

[1] R. Arnowitt and P. Nath, Spontaneous symmetry breaking of gauge supersymmetry, Phys. Rev. Lett. 36 (1976), no. 26, 1526-1529.

[2] D. Bleecker, Gauge Theory and Variational Principles, Global Analysis Pure and Applied Series A, vol. 1, Addison-Wesley, Massachusetts, 1981.

[3] S. B. Bradlow, Special metrics and stability for holomorphic bundles with global sections, J. Differential Geom. 33 (1991), no. 1, 169-213.

[4] K. Cahill, General internal gauge symmetry, Phys. Rev. D (3) 18 (1978), no. 8, 2930-2931.

[5] J. Dell, J. L. deLyra, and L. Smolin, Quantization of a gauge theory with independent metric and connection fields, Phys. Rev. D (3) 34 (1986), no. 10, 3012-3024.

[6] C. Dullemond, Quarks and gluons in a model with induced geometry, Gen. Relativity Gravitation 20 (1988), no. 10, 989-1005.

[7] R. O. Fulp and L. K. Norris, Splitting of the connection in gauge theories with broken symmetry, J. Math. Phys. 24 (1983), no. 7, 1871-1887.

[8] R. O. Fulp, L. K. Norris, and W. R. Davis, Conformal extension of gauge theories with spontaneously broken symmetry, Phys. Rev. D 36 (1987), no. 4, 1220-1228.

[9] L. O'Raifeartaigh, Hidden gauge symmetry, Rep. Progr. Phys. 42 (1979), no. 1, 159-223.

[10] R. Percacci, Geometry of Nonlinear Field Theories, World Scientific, Singapore, 1986.

[11] L. H. Ryder, Quantum Field Theory, Cambridge University Press, Cambridge, 1985.

[12] A. Talmadge, A geometric formulation of the Higgs mechanism, J. Math. Phys. 33 (1992), no. 5, 1864-1868.

[13] A. Trautman, The geometry of gauge fields, Czechoslovak J. Phys. B 29 (1979), no. 1, 107-116.

Andrew Talmadge: Department of Curriculum and Instruction, College of Education and Human Development, University of New Orleans, New Orleans, LA 70148, USA

E-mail address: atalmadg@uno.edu 


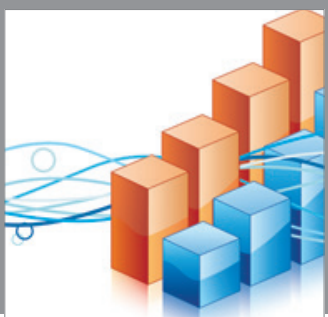

Advances in

Operations Research

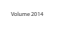

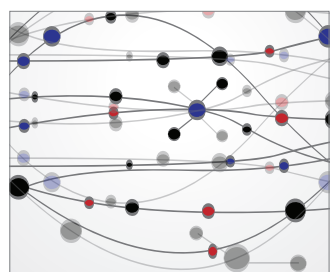

\section{The Scientific} World Journal
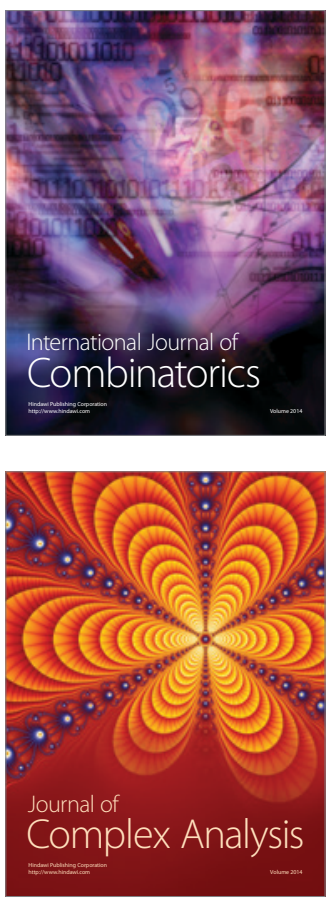

International Journal of

Mathematics and

Mathematical

Sciences
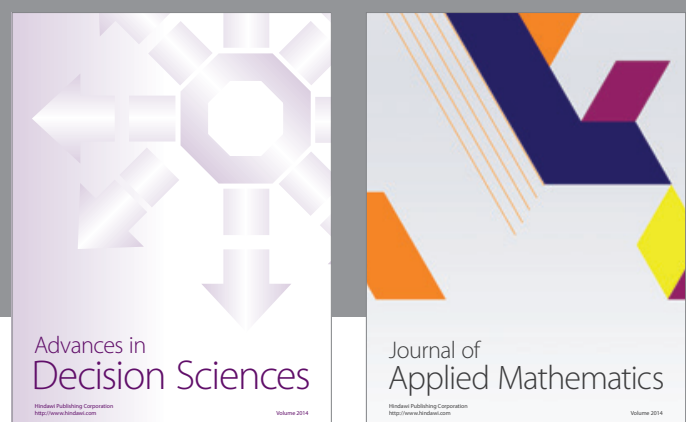

Journal of

Applied Mathematics
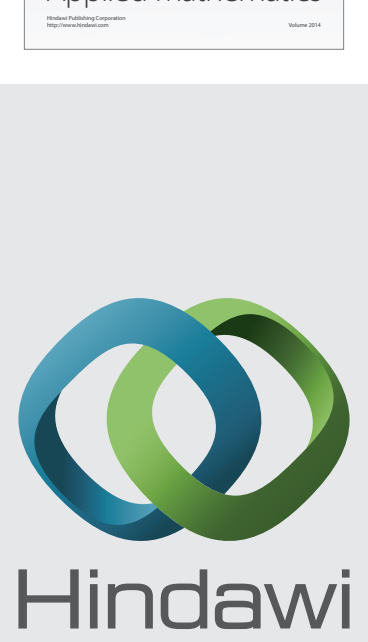

Submit your manuscripts at http://www.hindawi.com
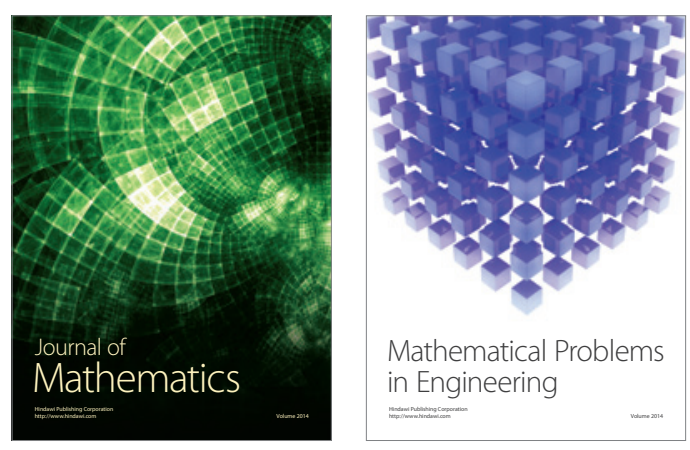

Mathematical Problems in Engineering
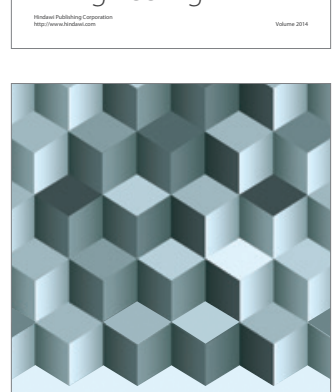

Journal of

Function Spaces
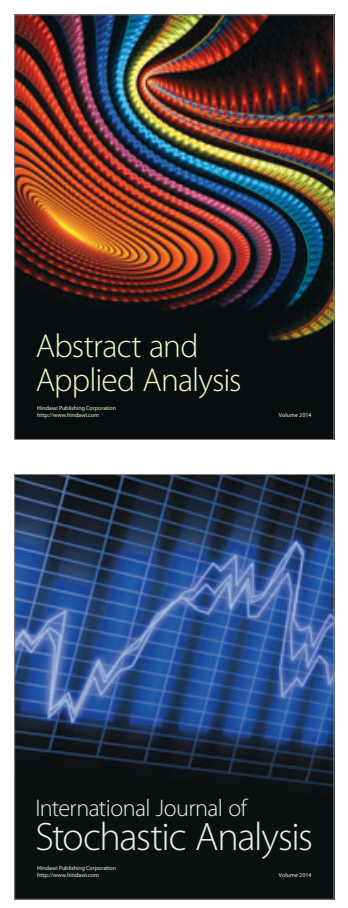

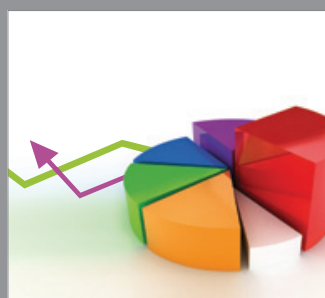

ournal of

Probability and Statistics

Promensencen
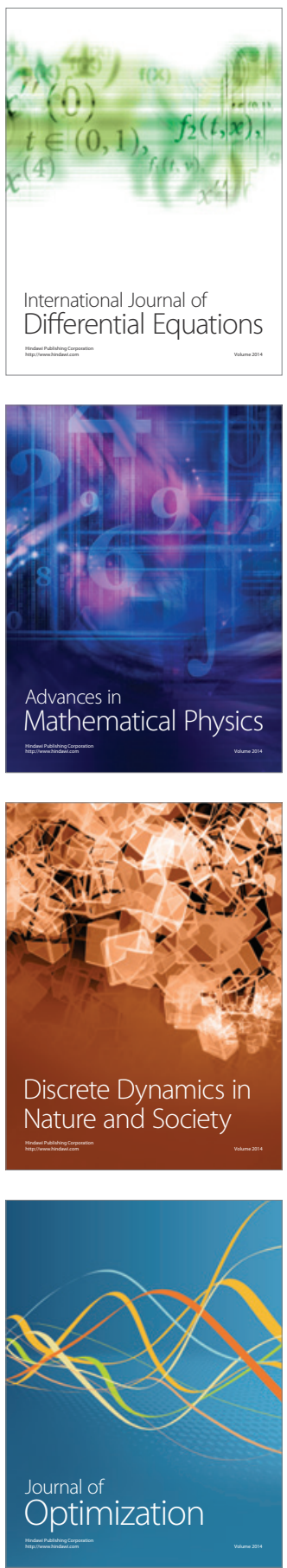